\title{
Pharmacists' knowledge of the safety of antibiotics for systemic use
}

\author{
Y. Yakimova* \\ Saratov State Medical University, Named after V.I. Razumovsky, Saratov, Russia \\ *Corresponding author. E-mail: yakimovayulia@gmail.com
}

BACKGROUND: Patients decide to take antibiotics themselves in $21 \%$ of cases of acute respiratory viral infections, influenza and acute respiratory infections [1]. The main factor of drug purchase at a pharmacy is pharmacist's recommendation. In $14 \%$ of cases of purchase of antibiotics, patients describe the symptoms and do not name a specific drug. This provides opportunity for drug selection at a pharmacy [2]. In these circumstances the role of pharmacists in ensuring the rational use of antimicrobial agents increases significantly.

OBJECTIVE: To evaluate the knowledge of pharmacists about antibiotics for systemic use.

METHODS: Pharmacoepidemiological study was based on surveying pharmaceutical workers using a questionnaire. The questionnaire included 2 groups of questions: general questions aimed at identifying the socio-demographic characteristics of respondents (gender, age) and professional status (level of qualification, work experience), as well as specific questions aimed at identifying perceptions and knowledge of respondents about the studied group of drugs (range of used drugs, the factors determining and limiting the choice of drug, properties of individual drugs, etc.). The study involved 182 pharmaceutical workers at the age of 20 to 52 years. When processing the received data, we used Microsoft Excel and BioStat, and methods of nonparametric statistics $\left(\chi^{2}\right.$-test). The survey was conducted anonymously, informed consent of the participants was not required. According to the recommendations of Ethical Committee of Saratov State Medical University n.a. V.I. Razumovsky (protocol No. 8 from 01.04.2014) the study is consistent with the basics of medical ethics.

RESULTS: Interviewed pharmaceutical workers were mostly women (97\%) with secondary pharmaceutical education (84\%). The average age of the respondents was $27.0 \pm 0.5$ years. The work experience of the respondents ranged from 0.5 to 34 years (average experience $5.64 \pm 0.41$ years).

Almost all pharmacists (97\%) reported that they dispense antimicrobial drugs every day and consult patients about the choice and characteristics of antibiotic (99.5\%). However, only $20 \%$ of the respondents indicated that they refuse to recommend antibiotics to the pharmacy visitors and send them to the doctor. Most often pharmaceutical employees recommended azithromycin (45\%), amoxicillin in combination with clavulanic acid (41\%), amoxicillin (26\%). According to respondents, the choice of recommended antibiotic mostly depends (score on a scale of 1-5) on pharmacological characteristics of the drug: the effectiveness (of $4.66 \pm 0.04$ ) and safety $(4.16 \pm 0.07$ ). Thus, pharmaceutical specialist should be knowledgeable about characteristics of antimicrobial agents to conduct proper consultation.

According to the results of self-assessment of respondents' knowledge about antibiotics it is at an average level. $36.3 \%$ of respondents state that they are familiar with the range and features of most of the drugs. $41.8 \%$ of professionals are familiar with the range of the most popular drugs and their 
main features. Only $16.5 \%$ of respondents assess their knowledge about antibiotics at the highest level, indicating that they are fully familiar with the range and features of the drugs.

Aiming at identifying knowledge about the side effects of antibiotics pharmacists were asked to compare the most commonly prescribed drugs and their respective side effects, to position the drugs and groups of drugs in the range according to the degree of toxicity and to indicate which antibiotics can be used during pregnancy. Knowledge of pharmaceutical workers is mainly limited to the perceptions of non-specific side reactions that can occur when taking any antibiotic (dyspepsia, allergic reactions, headache, candidiasis). $54.9 \%$ of respondents rightly pointed out nonspecific reactions for all 7 mentioned drug, for individual drugs the proportion of correct answers varied from $65,4 \%$ (linezolid) to $81,3 \%$ (amoxicillin+clavulanic acid). The analysis revealed no relationship between respondents' answers and their qualifications $\left(\chi^{2}=0,053 ; p=0,818\right)$, work experience $\left(\chi^{2}=6,956 ; p=0,096\right)$ and selfassessed knowledge about antimicrobial drugs $\left(\chi^{2}=1,272 ; p=1,000\right)$. There were no respondents who correctly reported specific adverse reactions (hearing disorder for azithromycin, hemopoiesis oppression for linezolid, etc.) for each antibiotic. The proportion of correct answers ranged from $6,0 \%$ for the combination amoxicillin+clavulanic acid to $43,4 \%$ for cefixime and doxycycline. The relationship between knowledge of respondents and frequency of recommendations of the same group of drugs could not be determined.

Pharmacists assume that the safest antibiotics are macrolides (average rank place of 2,99 $\pm 0,15$ of 8), cephalosporins $(3,12 \pm 0,15)$ and penicillins $(3,38 \pm 0,22)$, the most toxic are tetracyclines $(5,61 \pm 0,17)$. It should be noted that average rank places are quite similar, which means serious differences of opinion between the specialists. When assessing the toxicity of individual drugs a combination of amoxicillin+clavulanic acid (average rank place of $2,92 \pm 0,29$ of 15) and ampicillin (of 4,88 $\pm 0,36$ ) were considered as the safest, the most toxic were tetracycline $(10,13 \pm 0,30)$ and gentamicin $(10,14 \pm 0,33)$. These perceptions are generally consistent with the views on the safety of antibiotics in outpatient practice [3].

Half of the respondents $(51,1 \%)$ correctly identified the antibiotics that can be used during pregnancy (FDA category B). $45,1 \%$ of respondents said that no antibiotics can be used during pregnancy. Only $2,7 \%$ of respondents named drugs contraindicated during pregnancy (category $\mathrm{C}$ and $\mathrm{D}$ ) - gentamicin, doxycycline, ciprofloxacin.

CONCLUSIONS: The study identified gaps in the knowledge of pharmacists about the safety of antimicrobial agents. Views of professionals about antibiotics are mostly consistent with current data about the properties of drugs. However, detailed analysis shows that such views are not supported by clear knowledge of the properties of each drug and are mostly intuitive. In terms of the prevalence of self-medication with antibiotics and OTC dispensing of antimicrobial drugs it is necessary to improve the professional knowledge of pharmaceutical workers on antibiotics for systemic use.

Keywords: Antibiotics, safety, knowledge, pharmacists, pharmacoepidemiology

Conflict of interest statement: There was no conflict of interests.

\section{References}

[1] How do the Russians care about their health? [Internet]. Profi Online Research; 2009 [updated 20 Feb 2009; cited 27 Aug 2015]. Available from: http://profiresearch.ru/files/pr200209.pdf

[2] Increasing role of pharmacist in drug consumers' choice [Internet]. Synovate Comcon Healthcare; 2014 [updated 27 Aug 2015; cited 27 Aug 2015]. Available from: http://www.synovate.ru/default.asp?artID=2955

[3] Stetsiouk OU, Andreeva IV, Kolosov AV, Kozlov RS. Safety and Efficacy of Antibiotics Used in Outpatient Setting. Clinincal Microbiology and Antimicrobial Chemotherapy. 2011;13(1):67-84 